Mathematical Modelling and Analysis

Volume 11 Number 4, 2006, PAGes 357-364

(c) 2006 Technika ISSN 1392-6292 print, ISSN 1648-3510 online

\title{
VORTICES ON A SPHERE
}

\section{G. ALOBAIDI ${ }^{1}$, M.C. HASLAM ${ }^{2}$ and R. MALLIER ${ }^{3}$}

${ }^{1}$ Department of Mathematics and Statistics, American University of Sharjah Sharjah,United Arab Emirates

E-mail: galobaidi@yahoo.ca

${ }^{2}$ Department of Mathematics and Statistics York University 4700 Keele Street, Toronto, Ontario M3J 1P3 Canada

E-mail: mchaslam@mathstat.yorku.ca

${ }^{3}$ Department of Applied Mathematics, University of Western Ontario London ON N6A 5B7 Canada

E-mail: rolandmallier@hotmail.com

Received September 4, 2005; revised October 8, 2006; published online December 15, 2006

Abstract. We consider flows on a spherical surface and use a streamfunction formulation to derive a nonlinear equation governing steady-state flows in that geometry. We present some new vortex solutions to this equation.

Key words: flows on a sphere, streamfunction, vortex

\section{Introduction}

In two-dimensional hydrodynamics, the equation governing a motion of an inviscid incompressible fluid can be written in terms of a streamfunction $\psi(x, y, t)$ as

$$
\nabla^{2} \psi_{t}-\frac{\partial\left(\psi, \nabla^{2} \psi\right)}{\partial(x, y)}=0
$$

where

$$
\frac{\partial(a, b)}{\partial(x, y)}=a_{x} b_{y}-a_{y} b_{x}
$$

is a Jacobian and $\nabla^{2}=\frac{\partial^{2}}{\partial x^{2}}+\frac{\partial^{2}}{\partial y^{2}}$ is the two-dimensional Laplacian. This equation admits steady-state solutions of the form

$$
\nabla^{2} \psi=\mathcal{F}
$$


for any function $\mathcal{F}$. A number of solutions are known for plane two-dimensional hydrodynamics, including two solutions which represent rows of vortices: corotating Stuart vortices [11]

$$
\psi=\log [\cosh y-\varepsilon \cos x],
$$

with $\nabla^{2} \psi=\left(1-\varepsilon^{2}\right) e^{2 \psi}$, and counter-rotating Mallier-Maslowe (M\&M) vortices [9]

$$
\psi=\log \left[\frac{\cosh \varepsilon y-\varepsilon \cos x}{\cosh \varepsilon y+\varepsilon \cos x}\right],
$$

with $\nabla^{2} \psi=-\frac{1}{2}\left(1-\varepsilon^{2}\right) \sinh 2 \psi$.

\section{Vortices on a Sphere}

In the present paper, we are interested in the counterpart of equation (1.1) for a spherical surface. In particular we want to find steady-state vortex solutions on such a surface. In what follows, we will work in spherical coordinates $(r, \theta, \phi)$, so that the surface is defined by $0 \leq \theta \leq 2 \pi, 0 \leq \phi \leq \pi$, while the radial distance $r$ is constant.

In this coordinate system, we write the velocity as $\underline{u}=(u, v, w)$ with $u=0$, since there is no radial motion. Let us introduce a streamfunction via $v=\psi_{\phi} \csc \theta$ and $w=-\psi_{\theta}$. Then the inviscid incompressible equations of motion become

$$
\Omega_{t}-\frac{1}{r \sin \theta} \frac{\partial(\psi, \Omega)}{\partial(\theta, \phi)}=0
$$

together with a radial pressure gradient $p_{r}=r^{-1}\left[\psi_{\phi}^{2} \csc ^{2} \theta+\psi_{\theta}^{2}\right]$. In equation (2.1) we denote $\Omega=r^{2} \nabla^{2} \psi$, where

$$
r^{2} \nabla^{2} \equiv \csc ^{2} \theta \frac{\partial^{2}}{\partial \phi^{2}}+\cot \theta \frac{\partial}{\partial \theta}+\frac{\partial^{2}}{\partial \theta^{2}}
$$

is the Laplace-Beltrami operator. The equation (2.1) admits steady-state solutions of the form

$$
\Omega=\mathcal{F}(\psi) .
$$

This formulation is not new, and has been used by a number of authors to study vortices on a sphere. However, very few exact smooth solutions to this equation are known for flow on a sphere. Crowdy's generalization of the Stuart vortices to the sphere [2] is the most recent, and possibly the most substantial. Crowdy solved the counterpart of Liouville's equation on a sphere, $\Omega=a e^{b \psi}+c$, using a stereographic projection, which according to Lamb [8] was used by Kirchoff in 1875 to study electrical conduction in a spherical sheet. According to Crowdy, the only other exact distributed vortex equilibria (as 
opposed to point vortices) on a sphere known to him were the exact solutions on a rotating sphere presented in $[12,13]$ and Crowdy's own work [3] involving a combination of vortex patches and point vortices.

The bulk of the research to date on vortices on a sphere can be split into two main threads. The first thread, point vortices on a sphere, has a long history, with Lamb [8] discussing how some of the 19th century work on electrical conduction by Boltzmann, Kirchhoff, Töpler and others could be applied to this problem. More recent works are given by $[1,4,7]$ and the work on streets of point vortices is presented by [6].

The second thread involves using numerical methods, such as contour surgery, to study the motion of vortex patches on a sphere, which was pioneered by Dritschel and co-workers $[4,5,10]$. Although such analysis has revealed the richness of vortex motion on a sphere, the results were numerical rather than closed form expression.

Returning to $(2.2)$, we will work directly with this equation rather than using a projection. If we assume that $\psi=G(\sin \theta \sin \tilde{\phi})$, where $\tilde{\phi}=\phi+\phi_{0}$ and $\phi_{0}$ is a constant, then we find

$$
\Omega=\left(1-\sin ^{2} \theta \sin ^{2} \tilde{\phi}\right) G^{\prime \prime}(\sin \theta \sin \tilde{\phi})-2 \sin \theta \sin \tilde{\phi} G^{\prime}(\sin \theta \sin \tilde{\phi}) .
$$

We see that $\Omega$ is also a function of $\sin \theta \sin \tilde{\phi}$, so that $\psi=G(\sin \theta \sin \tilde{\phi})$ is a solution for any function $G$, and streamlines are lines on the sphere for which $\sin \theta \sin \tilde{\phi}$ is constant.

When $\phi_{0}=0$, this solution is trivial, because in spherical coordinates, $\sin \theta \sin \phi=y / r$, so the streamlines are lines on the sphere for which $y$ is constant, and the flow is symmetric about the $y$ axis. One example of this is given by

$$
\begin{aligned}
& \psi=\ln \left[1+\varepsilon(\sin \theta \sin \tilde{\phi})^{n}\right], \\
& \Omega=n e^{-2 \psi}\left(e^{\psi}-1\right)\left[\left(n-e^{\psi}\right)\left(\frac{\varepsilon}{e^{\psi}-1}\right)^{2 / n}-\left(n+e^{\psi}\right)\right],
\end{aligned}
$$

for $n$ a positive integer, with the velocity components given by

$$
(u, v, w)=\frac{n \varepsilon(\sin \theta \sin \tilde{\phi})^{n}}{\sin \theta\left[1+\varepsilon(\sin \theta \sin \tilde{\phi})^{n}\right]}(0, \cot \tilde{\phi},-\cos \theta)
$$

and the pressure gradient given by

$$
p_{r}=\frac{n^{2} \varepsilon^{2}(\sin \theta \sin \tilde{\phi})^{2 n-2}\left(1-\sin ^{2} \theta \sin ^{2} \tilde{\phi}\right)}{r\left[1+\varepsilon(\sin \theta \sin \tilde{\phi})^{n}\right]^{2}} .
$$

For the case $n=1$, we recover $\Omega=\left(1-\varepsilon^{2}\right) e^{-2 \psi}-1$, the counterpart of Liouville's equation, while for $n=2$, we have 


$$
\Omega=2(1+\varepsilon)\left(2 e^{-2 \psi}-e^{-\psi}\right)-2 .
$$

Two particular values of $\phi_{0}$ are special cases. For $\phi_{0}=0, w$ is zero at the poles for all $n$, while $v=0$ at the poles for $n>1$, but for $n=1, v=\varepsilon$ at $\phi=0$ and $v=-\varepsilon$ at $\phi=\pi$. At the equator, $\phi=\pi / 2, v=0$ for all $n$, while

$$
w=-\frac{n \varepsilon \sin ^{n-1} \theta \cos \theta}{1+\varepsilon \sin ^{n} \theta} .
$$

As discussed above, this solution is in a sense trivial, because the flow is symmetric about the $y$ axis. A similar, but less trivial, case is $\phi_{0}=\pi / 2$, so that $\sin \tilde{\phi}=\cos \phi$, where $v$ is zero at the poles for all $n$, but

$$
w= \begin{cases}-\frac{n \varepsilon \sin ^{n-1} \theta \cos \theta}{1+\varepsilon \sin ^{n} \theta}, & \phi=0, \\ -\frac{(-1)^{n} n \varepsilon \sin ^{n-1} \theta \cos \theta}{1+(-1)^{n} \varepsilon \sin ^{n} \theta}, & \phi=\pi .\end{cases}
$$

At the equator, $w=0$ for all $n$, while $v=-\varepsilon$ for $n=1$ and $v=0$ for $n \geq 2$.

Another vortex solution is defined as

$$
\begin{aligned}
\psi & =\ln \left[\frac{1+\varepsilon(\sin \theta \sin \tilde{\phi})^{n}}{1-\varepsilon(\sin \theta \sin \tilde{\phi})^{n}}\right] \\
\Omega & =\frac{n}{4}\left(e^{-2 \psi}-1\right)\left[n e^{2 \psi}+2 e^{\psi}+n-\left(n e^{2 \psi}-2 e^{\psi}+n\right)\left(\varepsilon \tanh \frac{\psi}{2}\right)^{2 / n}\right] .
\end{aligned}
$$

For $n=1$, we get a form of the double sinh Poisson equation

$$
\Omega=-\frac{1}{2}\left(1-\varepsilon^{2}\right)(2 \sinh \psi+\sinh 2 \psi),
$$

while for $n=2$,

$$
\Omega=e^{-2 \psi}\left[(\varepsilon-1) e^{3 \psi}+\varepsilon+1\right]\left(1+e^{\psi}\right) .
$$

The velocity components are given by

$$
(u, v, w)=\frac{2 n \varepsilon(\sin \theta \sin \tilde{\phi})^{n}}{\sin \theta\left[1-\varepsilon^{2}(\sin \theta \sin \tilde{\phi})^{2 n}\right]}(0, \cot \tilde{\phi},-\cos \theta)
$$

and the pressure gradient given by

$$
p_{r}=\frac{4 n^{2} \varepsilon^{2}\left(1-\sin ^{2} \theta \sin ^{2} \tilde{\phi}\right)(\sin \theta \sin \tilde{\phi})^{2 n-2}}{r\left[1-\varepsilon^{2}(\sin \theta \sin \tilde{\phi})^{2 n}\right]^{2}} .
$$


For the special case $\phi_{0}=0, w$ is zero at the poles for all $n$, while $v=0$ at the poles for $n>1$, but for $n=1, v=2 \varepsilon$ at $\phi=0$ and $v=-2 \varepsilon$ at $\phi=\pi$. At the equator, $v=0$ for all $n$, while

$$
w=-\frac{2 n \varepsilon \sin ^{n-1} \theta \cos \theta}{1-\varepsilon^{2} \sin ^{2 n} \theta} .
$$

Once again, this solution is in a sense trivial. For the case $\phi_{0}=\pi / 2, v$ is zero at the poles for all $n$, but

$$
w= \begin{cases}-\frac{2 n \varepsilon \sin ^{n-1} \theta \cos \theta}{1-\varepsilon^{2} \sin ^{2 n} \theta}, & \phi=0, \\ -\frac{(-1)^{n} 2 n \varepsilon \sin ^{n-1} \theta \cos \theta}{1-\varepsilon^{2} \sin ^{2 n} \theta}, & \phi=\pi .\end{cases}
$$

At the equator, $w=0$ for all $n$, while $v=-2 \varepsilon$ for $n=1$ and 0 for $n \geq 2$.

\section{Examples}

The streamlines for these vortex solutions (2.3), (2.4) coincide, and correspond to $\sin \theta \sin \tilde{\phi}=$ const, they are plotted in Figures $1-3$.

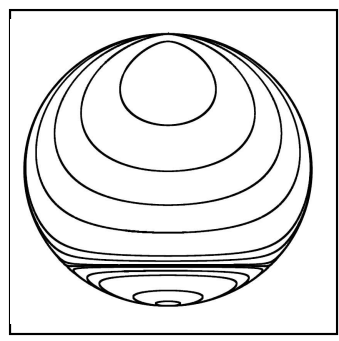

a)

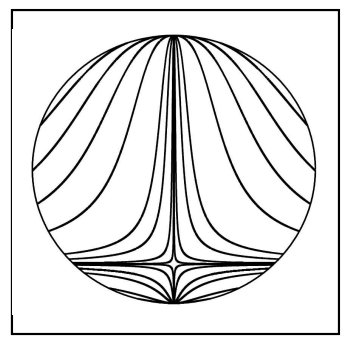

b)

Figure 1. Vortices with $\phi_{0}=\pi / 4$ as seen (a) from the $y$-axis; (b) from the $x$-axis.

Figure 1 shows a general case with $\phi_{0}=\pi / 4$, while Figures 2 and 3 show the special cases $\phi_{0}=0$, for which the streamlines are circles centered on the $y$ axis, and $\pi / 2$ respectively. In these figures, the figure labelled (a) shows the streamlines seen from the positive $y$ axis, that labelled (b) shows the streamlines seen from the positive $x$ axis, and Figure 3(c) shows the view from the positive $z$ axis. Figure 3(c) bears something of a resemblance to Figure 2 (a) of [4], although they are seen from different viewpoints. These figures illustrate that for the general case, the solutions (2.3), (2.4) represent a pair of vortices in each of the northern and southern hemispheres. When $\phi_{0}=0$, each of the northern hemisphere vortices merges with its counterpart in the southern hemisphere, so that we have two vortices centered on the equator. 


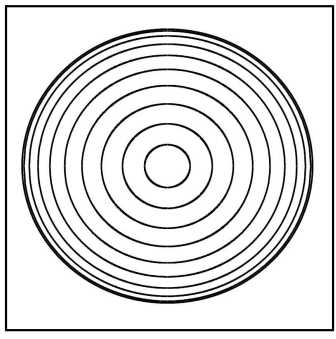

a)

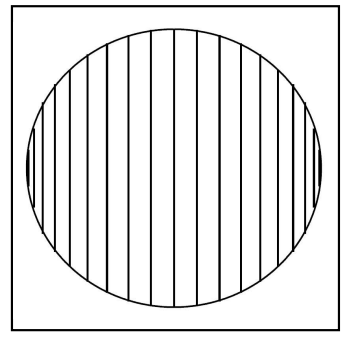

b)

Figure 2. Vortices with $\phi_{0}=0$ as seen (a) from the $y$-axis; (b) from the $x$-axis.

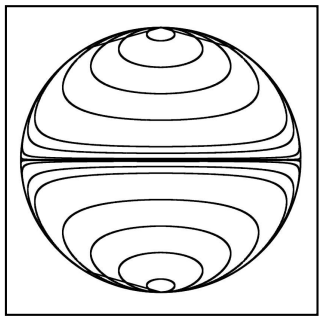

a)

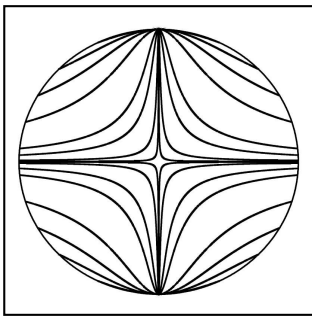

b)

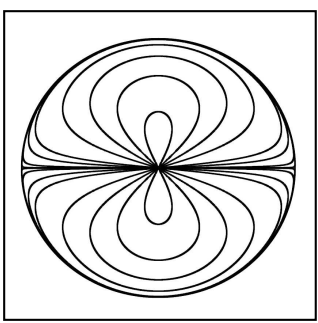

c)

Figure 3. Vortices with $\phi_{0}=\pi / 2$ as seen (a) from the $y$-axis; (b) from the $x$-axis; (c) from the $z$-axis.

When $\phi_{0}=\pi / 2$, the northern hemisphere vortices come together to produce a dipole-like structure, as do the southern hemisphere vortices.

Although the streamlines of solutions (2.3), (2.4) coincide, the streamfunction will take different values on the streamlines in the two cases, and different values for different $n$. In Figure 4 , we plot $\Omega$ on the line $\phi=\pi / 2$, or equivalently $z=0$, for several values of $n$, taking $\varepsilon=0.25$. (2.3) and (2.4) are plotted in Figure 4 (a) and (b) respectively for $\phi_{0}=0$ and in Figure 4 (c) and (d) for $\phi_{0}=\pi / 2$.

Solutions (2.3), (2.4) to nonlinear equation (2.2) presented here are just two specific examples, and it is likely that a number of different exact nonlinear vortex solutions exist, just as they do in the plane two-dimensional case. Indeed, this is strongly suggested by the numerical results presented in $[4,5,10]$, where various vortex flows were studied. 


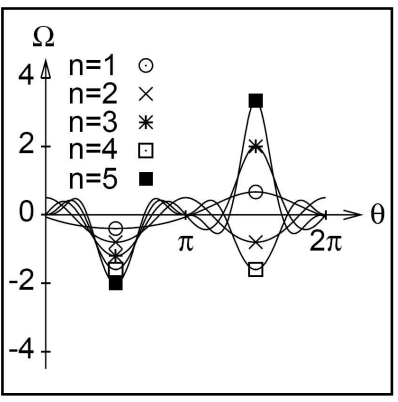

a)

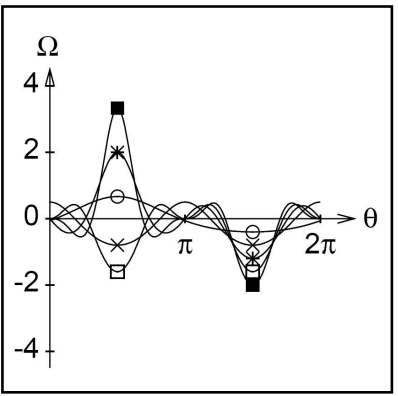

c)

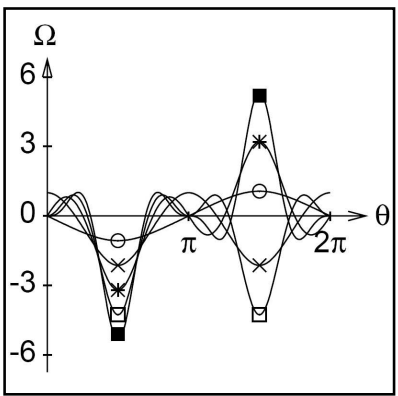

b)

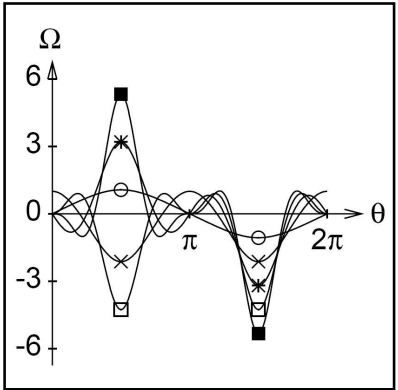

d)

Figure 4. $\Omega=r^{2} \nabla^{2} \psi$ along the line $\phi=\pi / 2$. (a) (2.3) with $\phi_{0}=0$; (b) (2.4) with $\phi_{0}=0 ;$ (c) (2.3) with $\phi_{0}=\pi / 2$; (d) (2.4) with $\phi_{0}=\pi / 2$.

\section{References}

[1] V.A. Bogomolov. Dynamics of vorticity on a sphere. Fluid Dyn., 6(863), 1977.

[2] D.G. Crowdy. Stuart vortices on a sphere. J. Fluid Mech., 498, 381-402, 2004.

[3] D.G. Crowdy and M. Cloke. Analytical solutions for distributed multipolar vortex equilibria on a sphere. Phys. Fluids, 15, 22-34, 2003.

[4] M.T. Dibattista and L.M. Polvani. Barotropic vortex pairs on a rotating sphere. Journal of Fluid Mechanics, 358, 107-133, 1998.

[5] D.G. Dritschel. Contour dynamics/surgery on the sphere. J. Comp. Phys., 79, 477-483, 1988.

[6] D. Hally. Stability of streets of vortices on surfaces of revolution with a reflection symmetry. J. Math. Phys., 21, 211-217, 1980.

[7] R. Kidambi and P.K. Newton. Point vortex motion on a sphere with solid boundaries. Phys. Fluids, 12, 581-588, 2000.

[8] H. Lamb. Hydrodynamics, 6th edition. Cambridge, 1932.

[9] R. Mallier and S.A. Maslowe. A row of counter rotating vortices. Phys. Fluids, A 5, 1074-1075, 1993.

[10] L.M. Polvani and D.G. Dritschel. Wave and vortex dynamics on the surface of a sphere. Journal of Fluid Mechanics, 225, 35-64, 1993.

[11] J.T. Stuart. On finite amplitude oscillations in laminar mixing layers. Journal of Fluid Mechanics, 29, 417-440, 1967.

[12] W.T.M. Verkley. The construction of barotropic modons on a sphere. J. Atmos. Sci, 41, 2492-2504, 1984. 
[13] W.T.M. Verkley. Nonlinear structures with multivalued $(q, \theta)$ relationshipsexact solutions of the barotropic vorticity equation on a sphere. Geophys. Astrophys. Fluid Dyn., 69, 77-94, 1993. 